\title{
Absorption and Scattering Cross-Section Extinction Values of Silver Nanoparticles
}

May Hlaing ${ }^{1}$, Bellsabel Gebear-Eigzabher ${ }^{2}$, Azael Roa ${ }^{1}$, Aristides Marcano*², Daniela Radu ${ }^{2,3}$, and Cheng-Yu Lai ${ }^{2}$

${ }^{1}$ Department of Physics and Engineering, Delaware State University, 1200 North DuPont Highway, Dover, DE 19901

${ }^{2}$ Department of Chemistry, Delaware State University, 1200 North DuPont Highway, Dover, DE 19901

${ }^{3}$ Department of Materials Science and Engineering, University of Delaware, Newark, DE, 19716 Abstract
We determine the extinction values of silver nanoparticles as a function of their diameter for
three different wavelengths ( $405 \mathrm{~nm}, 532 \mathrm{~nm}$, and $671 \mathrm{~nm}$ ) from the values of absorbance and
their photothermal lens response. We show that for particles of small diameters ( $<50 \mathrm{~nm})$ the
extinction grows as the cube of the diameter for all three wavelengths. For larger particles the
extinction determined from absorbance exhibits a sixth order dependence on the diameters for
$532 \mathrm{~nm}$ and $671 \mathrm{~nm}$. This kind of behavior is typical of scattering processes that should dominate
for large particles. For $405 \mathrm{~nm}$ the plasmonic resonant absorption dominates over scattering
making difficult the observation of the sixth order dependence even for particles larger than 50
$\mathrm{~nm}$. The absorption cross-section measured by the photothermal method does not show the sixth
order dependence. It depends on the cube of the particle's diameter for all nanoparticles
confirming the scattering free character of this absorption technique and validating the results of
the absorbance experiment.

* Corresponding author. Email: amarcano@desu.edu

Keywords: Silver nanoparticles, extinction cross-section, photothermal methods. 


\section{Highlights}

- Absorbance and photothermal methods are used to characterize silver nanoparticles.

- Absorption and scattering cross sections of silver nanoparticles are determined.

- Absorbance cross section increases as the cube of the particle's diameter.

- Scattering cross section is proportional to the sixth order of the diameter. 


\section{Introduction}

Silver nanoparticles (Ag NPs) have been the subject of numerous studies for many years becauseof their potential uses as bactericides and fungicides [1-4], as biological and chemical sensors [5-8], as conductive inks, as pastes and fillers, among other uses in molecular diagnostics, electronics, and photonics devices [7-10]. Despite the evident advances, determination of optical, electrical, and thermal properties of silver nanoparticles is still an open area of research. In this work we propose the combined use of regular absorption spectroscopy and photothermal lens (PTL) spectrometry for the determination of the extinction cross-section and the contribution of the absorption and scattering cross-sections values of Ag NPs of different dimensions. We show that absorption and scattering components of the extinction cross-section can be distinguished in a regular absorption experiment by their different dependence on the particle dimensions. According to the Rayleigh model, the absorption of one individual nanoparticle grows proportional to the third order of the particle's diameter while its scattering cross-section is proportional to the sixth order of the same magnitude [11-14]. We provide experimental evidence that such substantially different behaviors can be actually observed from the data obtained using regular absorbance spectroscopy. We show that these differences are remarkable for light wavelengths shifted toward the red, far from the region of plasmonic resonances, for which absorption dominates over the scattering effects. By fitting the experimental results based on this model we determine the contribution of the absorption and scattering effects to the total extinction cross-section values of an individual Ag NP.

We validate the results of the absorbance experiments using a PTL method which basically provides only the absorption contribution to the NPs extinction cross-section values [15-18]. Indeed, the PTL method measures the amount of heat released by the nanoparticles, 
following the absorption of light photons. In this regards, scattering effects cannot contribute. This particular property of the PTL methods has been demonstrated by different authors [19-23]. Furthermore, the combined use of absorbance and PTL method allows the determination of scattering and fluorescence quantum yields of the sample [24-26]. In this work, we use a pumpprobe PTL approach to determine the corresponding absorption cross-section of Ag NPs of different dimensions. As expected, we show that this contribution is proportional to the cube of the nanoparticle diameter. The sixth order dependence on the particle's dimensions was not observed in the PTL experiment. Comparison of the PTL and absorbance results allows validation and identification of absorption and scattering contribution to the total extinction value of the nanoparticle. Although this study is limited to Ag NPs, the method bears general character and can be applied for other nanoparticles.

\section{Theoretical Considerations}

\subsection{Determining absorption and scattering cross-sections based on absorbance spectroscopy.}

We consider that the loss of light energy upon propagation through the solution of nanoparticles results only in the generation of heat and scattering. In this situation, the extinction cross-section of the nanoparticle can be written as:

$$
\sigma(\lambda)=\sigma_{a b s}(\lambda)+\sigma_{s c a}(\lambda),
$$

where $\lambda$ is the light wavelength, and $\sigma_{a b s}$ and $\sigma_{s c a}$ are the absorption and scattering crosssections, respectively. For a nanoparticle with dimensions much smaller than the wavelength of the propagating light, we have [27]:

$$
\sigma_{a b s}(\lambda)=8 \pi^{2} \operatorname{Re}(i \alpha(\lambda)) / \lambda,
$$




$$
\sigma_{s c a}(\lambda)=128 \pi^{5}|\alpha(\lambda)|^{2} /\left(3 \lambda^{4}\right),
$$

Where $\alpha$ is the polarizability of the nanoparticle, and $i=\sqrt{-1}$. Lorentz formula provides the value of the polarizability $\alpha(\lambda)$ for a spherical particle of diameter $d$ as:

$$
\alpha(\lambda)=[(\varepsilon(\lambda)-1) /(\varepsilon(\lambda)+2)] d^{3} / 8,
$$

where $\varepsilon=\mathcal{E}^{\prime}-i \mathcal{E}^{\prime \prime}$ is the complex permittivity of the nanoparticle, and $\varepsilon^{\prime}$ and $\mathcal{E}^{\prime \prime}$ are its real and the imaginary part, respectively. Taking into account equations (2) through (4) the extinction crosssection can be written as:

$$
\sigma(\lambda)=B(\lambda) d^{3}+D(\lambda) d^{6},
$$

where:

$$
\begin{gathered}
B(\lambda)=\pi^{2} \operatorname{Re}[i(\varepsilon(\lambda)-1) /(\varepsilon(\lambda)+2)] / \lambda, \\
D(\lambda)=2 \pi^{5}|(\varepsilon(\lambda)-1) /(\varepsilon(\lambda)+2)|^{2} /\left(3 \lambda^{4}\right) .
\end{gathered}
$$

Equation 5 shows that the extinction cross-section is basically due to absorption for small nanoparticles. For large nanoparticles the scattering contribution becomes dominant. Experimental values of the extinction cross-sectionof the nanoparticles can be determined according to the Beer-Lambert law as

$$
\sigma(\lambda)=A(\lambda) \ln 10 / N L
$$

where $A(\lambda)$ is the absorbance expressed as a function of the light wavelength, $L$ is the pathlength of the sample, and $N$ is the concentration of nanoparticles. For a sample of spherical nanoparticles of diameter $d(m)$ and sample's concentration $C\left(\mathrm{Kg} / \mathrm{m}^{3}\right), N\left(\mathrm{~m}^{-3}\right)$ can be calculated from the formula:

$$
N=6 C /\left(\pi d^{3} \rho\right)
$$


where $\rho=10.510^{3} \mathrm{Kg} / \mathrm{m}^{3}$ is the density of silver. Based on the dependence of the extinction cross-section on the dimensions of the nanoparticles we estimate the values of the absorption and scattering extinction cross-sections using equations 1 and 5.

Determination of scattering cross-section using this method is limited when absorption dominates the interaction between the nanoparticle and the light. In this case, sixth order dependence on the particle diameter might not be observed. Another limitation is related to the dimensions of the particle. If the particle has a diameter of the order of the wavelength $(d \geq 200$ $\mathrm{nm})$ the penetration of light into the particle is not complete. In this case, we expect a reduction of the extinction cross-section because of the limited coupling between the light and the particle.

\subsection{Determining absorption cross-section using the PTL method.}

PTL experiment is an alternative way to study absorption. The method is based on the detection of the amount of heat released in the sample following the absorption of light photons. In a pump-probe PTL experiment, the light from a pump laser is focused onto the sample generating a local field of the refraction index of thermal origin or thermal lens (TL). The TL is then tested by a collimated probe beam of light which propagates collinearly to the pump beam. The presence of the TL distorts the wavefront of the probe beam inducing changes in its diffraction pattern at the far field. We measure the relative transmission of the probe light through a small aperture located at the far field.Thus, the PTL signal can be defined as:

$$
S(z, t)=\left(T(z, t)-T_{o}\right) / T_{o},
$$

where $T_{o}$ is the probe light transmission through the aperture in the absence of the pump field and $T(z, t)$ is the probe light transmission in the presence of the pump field. The signal has been written as a function of time $t$ and sample position $z$. We consider that the pump and the probe 
beam are Gaussians with Rayleigh ranges $z_{e}$ and $z_{p}$, and wavelengths $\lambda_{e}$ and $\lambda_{p}$, respectively. We also consider that the pump beam waist is at position $z=0$ and the probe beam waist is at position $z=a_{p}$. A Fresnel diffraction model shows that for small changes in probe light transmission $\left(\left|T(z, t)-T_{o}\right|<<T_{o}\right)$ the PTL signal is given by [18]:

$$
S(z, t)=S_{o} \tan ^{-1}\left(4 m(z) V(z)\left[t / t_{c}(z)\right]\left\{\left[1+2 m(z)+V^{2}(z)\right] 2 t / t_{c}(z)+[1+m(z)]^{2}+V^{2}(z)\right\}^{-1}\right),
$$

$$
\begin{gathered}
S_{o}=P_{e} \psi A L_{e f}(d n / d t) /\left(\kappa \lambda_{p}\right), \\
V(z)=\left(z-a_{p}\right) / z_{p}+\left[\left(z-a_{p}\right)^{2}+z_{p}^{2}\right]\left[z_{p}\left(d_{e}-z\right)\right]^{-1}, \\
m(z)=\lambda_{p} z_{p}\left[1+\left(z-a_{p}\right)^{2} / z_{p}^{2}\right]\left[\lambda_{e} z_{e}\left(1+z^{2} / z_{e}^{2}\right)\right]^{-1} \\
t_{c}(z)=\lambda_{e} z_{e}\left(1+z^{2} / z_{e}^{2}\right) /(4 \pi D),
\end{gathered}
$$

$P_{e}$ is the total power of the pump beam, $\psi$ is the fractional thermal load, $A$ is the sample's absorbance, $L_{e f}=[1-\exp (-A L)] / A, L$ is the sample's pathlength, $d n / d t$ is the thermal gradient of the refraction index, $\kappa$ is the sample's thermal conductivity, $d_{e}$ is the position of the detection plane, and $D$ is the sample's thermal diffusivity. To determine the value of the thermal load $\Psi$ we scan the sample in the longitudinal direction around the waist of the pump beam (Z-scan). The result is a signal peaked at the position of the pump beam waist. The fitting of the Z-scan signature using equation 11 allows determination of $S_{o}$. If the values of the absorbance $A$, photothermal parameters $d n / d T$, $\kappa$, and $D$, are known it becomes possible to determine $\Psi$ using equation 12. In the situation when the sample exhibits only absorption and scattering, the absorption and scattering cross-sections will be:

$$
\sigma_{a b s}(\lambda)=\Psi \cdot \sigma(\lambda)
$$




$$
\sigma_{s c a}(\lambda)=(1-\Psi) \cdot \sigma(\lambda)=\sigma(\lambda)-\sigma_{a b s}(\lambda),
$$

Because the PTL signal does not include the scattering contribution we expect that its dependence on the diameter should be of third order with no $6^{\text {th }}$ order component for any wavelength. Below we provide experimental confirmation of this fact.

\section{Experimental Method}

We studied silver nanoparticles solutions in water at the concentration of $0.02 \mathrm{mg} / \mathrm{ml}$ (NanoComposix). The samples were used without further purification. The diameters of the nanoparticles are 5, 10,20,30,50,70,80,100, and $200 \mathrm{~nm}$, respectively. We also used a sample of $0.65 \mathrm{~nm}$ diameter of the same concentration provided by Purest Colloids. We used a UV-VIS spectrophotometer (Evolution 201 Thermo-Scientific) to determine the absorbance spectra of each of the samples. To contain the samples we used standard 1-cm path-length quartz cells. Distilled deionized water was used as a reference. We selected the values of absorbance for each Ag NP sample for three wavelengths: $405 \mathrm{~nm}, 532 \mathrm{~nm}$, and $671 \mathrm{~nm}$, to study the dependence of the extinction cross-section on the particle's dimensions. The concentration of nanoparticles was estimated using equation 9. We determined the extinction cross-section using Eq. 8.

To perform PTL experiments we used a mode-mismatched experimental scheme where the pump beam is focused and the probe beam is collimated [18]. We conduct Z-scan PTL experiments at the same pump wavelengths $(405,532$ and $671 \mathrm{~nm})$ used for the analysis of the absorbance experiment. The experimental set-up is shown in figure 1. Diode lasers generating at 405, 532and671 $\mathrm{nm}$ provide the pump beam. The pump light is focused onto the sample (S) using a mirror $\left(\mathrm{M}_{1}\right)$ and a $15-\mathrm{cm}$ focal length lens $\left(\mathrm{L}_{1}\right)$ to generate the TL on it. A beamsplitter $\left(B_{1}\right)$ redirects a small amount of the pump light toward a reference detector (Ref). The reference 
detector is connected to a digital oscilloscope (OSC) for triggering purposes. A signal generator provides the modulation of the pump beam. We modulate the pump light at low frequencies $(0.1$ $2 \mathrm{~Hz}$ ) to make sure the signal reaches its stationary value. Behind the sample, the pump beam is depleted using an interference filter (F). A $2 \mathrm{~mW}$ He-Ne laser $(632 \mathrm{~nm})$ generates the probe beam. We use a telescope $(\mathrm{Col})$ to collimate the probe beam up to a diameter of $3 \mathrm{~mm}$. Considering the beams Gaussians we estimated the Rayleigh ranges for the pump and the probe beam as $z_{e}=0.7 \mathrm{~cm}$ and $z_{p}=100 \mathrm{~cm}$, respectively. We direct the probe beam collinearly to the pump beam using a beamsplitter $\left(\mathrm{B}_{2}\right)$. The TL distorts the wavefront of the probe beam. Behind the sample, the probe beam is redirected toward a small aperture (A) and then to a diode detector (Sig) which measures the transmission of the probe light through the aperture. The generated electronic signal is amplified using a current preamplifier (Stanford Research SR 570) and then is redirected to a digital oscilloscope (Tektronix TDS3052) for processing. For the PTL experiments, we used glass cells of $0.1 \mathrm{~cm}$ pathlength.

\section{Results and Analysis}

\subsection{Absorbance Results}

In figure 2 we show the absorbance spectra of Ag NPs of diameters: $5 \mathrm{~nm}$ (solid black line), $10 \mathrm{~nm}$ (solid red line), $20 \mathrm{~nm}$ (solid blue line), $50 \mathrm{~nm}$ (solid green line), $100 \mathrm{~nm}$ (solid magenta line), and $200 \mathrm{~nm}$ (solid olive line). NPs of a diameter smaller than $50 \mathrm{~nm}$ show a pronounced peak around $400 \mathrm{~nm}$. This peak corresponds to the plasmonic interaction between free electrons in the NPs which leads to resonant absorption of light of this part of the spectrum. The peak shifts toward red for larger nanoparticles. An additional broad peak appears which are understood as the results of higher multipole interaction between the NPs and light. Both, 
absorption and scattering contribute to the final shape of the absorbance spectra. By simple visualization of the absorbance spectrum shape, it is not possible to estimate the exact contribution of both processes. However, this estimation becomes possible by analyzing in details the dependence of the extinction cross-section values on the NPs' dimensions. In figure 3 the crossed black squares represent the extinction cross-section values $\left(\mathrm{m}^{2}\right)$ as a function of the NP's diameter for three different wavelengths: $405 \mathrm{~nm}$ (Fig. 3a), $532 \mathrm{~nm}$ (Fig. 3b), and $671 \mathrm{~nm}$ (Fig. 3c). The extinction cross-section values are calculated using Eq. (8) and the values of the absorbance obtained from these spectra. For determination of the concentration of NPs $\left(\mathrm{m}^{-3}\right)$ we use Eq. (9). For $405 \mathrm{~nm}$ we observe a clear third order dependence which indicates a strong plasmonic absorption and negligible scattering. The red solid line of figure $3 \mathrm{a}$ is calculated using Eq. 5 and parameters $B=(1.5 \pm 0.2) \cdot 10^{8} \cdot \mathrm{m}^{-1}$ and $D=0$. The dependence becomes smaller than the third order for NPs larger than $80 \mathrm{~nm}$. For larger NP's the absorption is expected to decrease due to the limited penetration of light into the NPs with dimensions close to the value of the light wavelength. For the wavelength $532 \mathrm{~nm}$, we record a third order dependence for NPs smaller on the value of particle's diameter $\mathrm{d}$ for $d<5010^{-9} \mathrm{~m}$ (see Fig. 3b). Larger NPs exhibit sixth order dependence which corresponds to a larger contribution of Rayleigh scattering. The red solid line is the fitting of the data using Eq. 5. From this fitting we obtain for the absorption and scattering cross-section of the Ag NPs the following empirical relations:

$$
\begin{aligned}
& \sigma_{a b s}(532 \quad n m)=(3 \pm 0.4) \cdot 10^{6} \cdot d^{3} \cdot m^{-1}, \\
& \sigma_{s c a}(532 \quad n m)=(4 \pm 0.5) \cdot 10^{28} \cdot d^{6} \cdot m^{-4},
\end{aligned}
$$

Where $d$ is expressed in meters. The results of the calculation of absorption cross-section using Eq. (18) is shown in figure $3 b$ by the blue dashed line. The result of the calculation of the scattering cross-sections using Eq. 19 is indicated by the green dashed line. We see that for $d<70$ 
$10^{-9} \mathrm{~m}$, the scattering contribution to the extinction is negligible. In this situation the fittings of the absorption (dash blue line) and total extinction (solid red line) superimpose. For $d>7010^{-9}$ $m$, the absorption contribution becomes small. In this situation, the fittings of the scattering contribution (green dashed line) and the total extinction (red solid line) superimpose. The results for $671 \mathrm{~nm}$ (Fig. 3c) show a more clear transition between third order and sixth order dependence. In this situation we obtain the empirical equations :

$$
\begin{aligned}
& \sigma_{a b s}(671 \mathrm{~nm})=(1 \pm 0.15) \cdot 10^{6} \cdot \mathrm{d}^{3} \cdot \mathrm{m}^{-1}, \\
& \sigma_{s c a}(671 \mathrm{~nm})=(6 \pm 0.7) \cdot 10^{27} \cdot \mathrm{d}^{6} \cdot \mathrm{m}^{-4}
\end{aligned}
$$

The results of the calculation for the absorption and scattering cross-sections are indicated by the blue dashed line and the green dashed line in Fig. 3c, respectively. Again the results show the dominance of absorption processes for NPs smaller than $50 \mathrm{~nm}$ and the dominance of Rayleigh scattering for larger NPs.

\subsection{PTL Results}

We perform PTL experiment as an alternative method for determination of the absorption cross-section. We perform a Z-scan experiment for each sample. The results of the Z-scan for 5 (crossed red squares), 50 (crossed blue circles), and $200 \mathrm{~nm}$ Ag NPs (pointed green stars) using a $20 \mathrm{~mW}$ of pump power at $532 \mathrm{~nm}$ are shown in figure 4. Similar results were obtained for $405 \mathrm{~nm}$ and $671 \mathrm{~nm}$. The Z-scan picture exhibits a single peak centered at the beam waist which magnitude is proportional to the sample's absorption. The Z-scan results are fitted using Eq. 11 and parameters $z_{e}=0.007 \mathrm{~m}, z_{p}=0.2 \mathrm{~m}, d_{e}=1 \mathrm{~m}, D=1.4110^{-7} \mathrm{~m}^{2} / \mathrm{s}, \mathrm{k}=$ and $S_{o}=0.128$ for $5 \mathrm{~nm} \mathrm{Ag}$ NPs, $S_{o}=0.076$ for $50 \mathrm{~nm} \mathrm{Ag} \mathrm{NPs,} \mathrm{and} S_{o}=0.0325$ for $200 \mathrm{~nm}$ Ag NPs. The diffusivity value corresponds to the diffusivity of pure water. The solids lines on the Z-scan curves show the 
results of the fitting for $5 \mathrm{~nm}$ (red solid line), $50 \mathrm{~nm}$ (blue solid line), and $200 \mathrm{~nm}$ (green solid line). By using the values $S_{o}$, the water parameters $d n / d T=10^{-4 o} K^{-1}, \quad \kappa=0.56 \mathrm{~W} \mathrm{~m}^{-1}{ }^{o} K^{-1}, L=0.001$ $m$, and the absorbance value we determine the thermal load values $\psi$ for each sample. The absorption cross-section $\sigma_{\text {abs }}$ can be then determined by Eq 16. A more practical approach for the calibration is considering that the absorption cross-section is equal to the extinction cross-section for small nanoparticles $\left(d<10^{-8} \mathrm{~m}\right)$ for which scattering contribution is expected to be at least two order of magnitude smaller than the absorption contribution. In Fig 5 we show the PTL results of the determination of the absorption cross-section as a function of the particle's diameter, for pump wavelengths $405 \mathrm{~nm}$ (crossed blue squares), $532 \mathrm{~nm}$ (solid green stars), and $671 \mathrm{~nm}$ (crossed red circles). For all three pump wavelengths used we observe a third order dependence on the particle dimension indicated by the black solid line in Fig. 5. This corresponds to an absorption effect with no scattering contribution. Our results are in agreement with previous reports on the scattering-free character of the PTL method [20-23]. For $405 \mathrm{~nm}$ we observe again a small deviation from the third order dependence toward lower values of the absorption for particles of dimensions larger than $80 \mathrm{~nm}$. This behavior confirms the results obtained in the absorbance experiment (see Fig. 3a).

\section{Conclusions}

We performed absorbance and PTL experiments to determine the extinction, absorption and scattering cross-section values of Ag NPs of different dimensions. We show that the extinction cross-section obtained from the absorbance experiment depends on the cube of the particle's diameter for particles of a diameter smaller than $70 \mathrm{~nm}$. For larger particles a sixth order dependence becomes evident in the spectral regions far from the plasmonic peak in the 
region of $400 \mathrm{~nm}$. The cross-section obtained using the PTL method confirms the absorption character of the signal without contribution from scattering effects. The results are in agreement with the basic Rayleigh model of absorption and scattering of small particles. Based on the observations we establish empirical equations for the determination of the absorption and scattering cross-section values of the nanoparticles.

\section{Acknowledgments}

We would like to thank the National Science Foundation (Grants $\mathrm{N}^{\mathrm{o}} 1242067, \mathrm{~N}^{\mathrm{o}}$ 1435716 , and $\mathrm{N}^{\mathrm{o}} 1535876$ ) for financial support of this research. 


\section{References}

[1] M. Bosetti, A. Masse, E. Tobin, M. Cannas, Silver coated materials for external fixation devices: in vitro biocompatibility and genotoxicity, Biomaterials 23 (2002) 887892.DOI:10.1016/S0142-9612(01)00198-3.

[2] A. Gupta, S. Silver, Molecular Genetics: Silver as a Biocide: Will resistance become a problem?, Nat. Biotechnol. 16 (1998) 888. DOI:10.1038/nbt1098-888.

[3] M. Cho, H. Chung, W. Choi, J. Yoon, Different Inactivation Behaviors of MS-2 Phage and Escherichia coli in $\mathrm{TiO}_{2}$ Photocatalytic Disinfection, Appl. Environ. Microbiol. 71 (2005) 270-275. DOI: 10.1128/AEM.71.1.270-275.200

[4] Yeo, S., Lee, H., Jeong, S., Preparation of nanocomposite fibers for permanent antibacterial effect, J. Mater. Sci. 38 (2003) 2143-2147.

[5] Manno, D., Filippo, E., Giulio, M., Serra, A., Synthesis and characterization of starchstabilized Ag nanostructures for sensors applications, 2008. J. Non-Cryst. Solids. 354 (2008) 5515-5520. DOI:10.1016/j.jnoncrysol.2008.04.059.

[6] Hahm, J., Lieber, C., "Direct Ultrasensitive Electrical Detection of DNA and DNA Sequence Variations Using Nanowire”, Nanosensors, 2004. Nano Lett. 4 (1), 51-54.DOI: 10.1021/n1034853b.

[7] J. Köhler, L. Abahmane, J. Albert, G. Mayer, Preparation of metal nanoparticles with varied composition for catalyticalapplications in microreactors, Chem. Eng. Sci. 63 (2008), 5048-5055. DOI:10.1016/j.ces.2007.11.038.

[8] J. Guo, H. Cui, W. Zhou, W. Wang, Ag nanoparticle-catalyzed chemiluminescent reaction between luminol and hydrogen peroxide, J. Photochem. Photobiol. A: Chem. 193 (2008) 89-96.DOI:10.1016/j.jphotochem.2007.04.034. 
[9] P. Liu, M. Zhao, Silver nanoparticle supported on halloysite nanotubes catalyzed reductionof 4-nitrophenol (4-NP), Appl. Surf. Sci. 255 (2009) 3989-3993.

[10] R. Jin, Y. Cao, C. Mirkin, K. Kelly, G. Schatz, J. Zheng, Photoinduced Conversion of Silver Nanospheres to Nanoprisms, Science 294(2001) 1901-1903. DOI: $10.1126 /$ science.1066541.

[11] Lord Rayleigh, On scattering of light by small particles, Phil. Mag., 41 (1871) 274279.

[12] Lord Rayleigh, On the transmission of light through an atmosphere containing small particles in suspension, and on the origin of the blue sky, Phil. Mag., 47 (1899) 375-394. [13] Lord Rayleigh, The incident of light upon a transparent sphere of dimensions comparable with the wavelength, Pro. Roy. Soc. A84 (1910) 25-46.

[14] K. Kelly, E. Coronado, L. Zhao, G. Schatz, The Optical Properties of Metal Nanoparticles: The Influence of Size, Shape, and Dielectric Environment, J. Phys. Chem. B 107 (2003) 668-677. DOI: 10.1021/jp026731y

[15] M.E. Long, R.L. Swofford, A.C. Albrecht, Thermal lens technique: A new method of Absorption Spectroscopy, Science 191(1976) 183-184.DOI:10.1126/science.1246605.

[16] R.D. Snook, R.D. Lowe, Thermal Lens Spectrometry: A Review, Analyst. 120(1995) 2051-2068.DOI:10.1039/AN9952002051.

[17] S. E. Bialkowski, Photothermal Spectroscopy Methods for Chemical Analysis, New York, Wiley 1996.

[18] A. Marcano, C. Loper, N. Melikechi, Pump-probe mode mismatched Z-scan, J. Opt. Soc. Am. B. 19 (2002) 119-124.DOI:10.1364/JOSAB.19.000119. 
[19] J.B. Thorne, D. Bobbitt. Comparison of Beer's law and thermal lens techniques for absorption measurements under conditions of high scattering backgrounds,Appl. Spectrosc. 47(1993) 360-365.

[20] J. Georges, Advantages and limitations of thermal lens spectrometry over conventional spectrophotometry for absorbance measurement, Talanta. 48(1999) 501509.DOI:10.1016/S0039-9140(98)00242-2.

[21] Z.A. Yasa, W.B. Jackson, N.M. Amer, Photothermal spectroscopy of scattering media, Appl. Opt. 21 (1982) 21-31. DOI:10.1364/AO.21.000021.

[22] A. Olivares, A. García-Valenzuela, F.L.S. Cuppo, F. Curiel, G. Ortiz and R.G. Barrera. Measurement of low optical absorption in highly scattering media using the thermal lens effect,J. Phys. IV 125 (2005) 153-156. DOI:10.1051/jp4:2005125035.

[23] A. Marcano, I. Basaldua, A. Villette, R. Edziah, J. Liu, O. Ziane, N. Melikechi,Photothermal lens spectrometry measurements in highly turbid media, Appl. Spectros. 67 (2013) 1013-1018. DOI: 10.1366/12-06970.

[24] A. Marcano, S. Alvarado, J. Meng, D. Caballero, E. Marin-Moares, R. EdziahWhite light photothermal lens spectrophotometer for determination of absorption in scattering samples, Appl. Spectros.68 (2014) 680-685. DOI: 10.1366/13-07385(2013).

[25] A. Marcano, J. Ojeda, N. Melikechi, Absorption spectra of dye solutions measured using a white-light thermal lens spectrophotometer, Appl. Spectros. 60 (5), 560-563 (2006).

[26] J. Hung, A. Marcano O., J. Castillo, J. Gonzalez, V. Piscitelli, A. Reyes, A. Fernandez, Thermal lensing and absorbance spectra of a fluorescent dye, Chem. Phys. Lett. 386 (2004) 206-210. 
[27] H.C. van de Hulst, "Light scattering by small particles", Dover Publications, Inc. N.Y.1981. 


\section{Figure Captions}

Figure 1. Experimental set-up for the PTL experiment consisting of a diode pump laser modulated by a signal generator, a probe $\mathrm{He}-\mathrm{Ne}$ laser, a collimator "Col", beamsplitters "B ${ }_{1}$ " and " $\mathrm{B}_{2}$ ", reference detector Ref, mirror $\mathrm{M}_{1}$ and $\mathrm{M}_{2}$, lens $\mathrm{L}$ 1, interference filter " $\mathrm{F}$ ", a sample $\mathrm{S}$, an aperture "A", a signal detector "Sig", a current amplifier "Amp" and a digital oscilloscope “Osc".

Figure 2. Absorbance spectra of the Ag NP of $5 \mathrm{~nm}$ (solid black line), $10 \mathrm{~nm}$ (solid red line), 20 $\mathrm{nm}$ (solid blue line), $50 \mathrm{~nm}$ (solid green line), $100 \mathrm{~nm}$ (solid magenta line), and $200 \mathrm{~nm}$ (solid olive line).

Figure 3. Extinction cross-section (crossed black squares) of the Ag NPs as a function of their diameter for excitation wavelength $405 \mathrm{~nm}$ (a), $532 \mathrm{~nm}$ (b) and $671 \mathrm{~nm}$ (c). In figure 3a the red solid line corresponds to a fitting using Eq. 5 and parameters $B=1.510^{8} \mathrm{~m}^{-1}$ and $D=0$. In figure $3 \mathrm{~b}$ the red solid line corresponds to a fitting using Eq. 5 and parameters $B=310^{6} \mathrm{~m}^{-1}$ and $D=4$ $10^{28} \mathrm{~m}^{-4}$, while the blue and green dashed lines are calculated using Eqs. 18 and 19, respectively. In figure $3 \mathrm{c}$ the red solid line corresponds to a fitting using Eq. 5 and parameters $B=10^{6} \mathrm{~m}^{-1}$ and $D=610^{27} \mathrm{~m}^{-4}$, while the dashed blue and green lines are calculated using Eqs. 20 and 21, respectively.

Figure 4. PTL Z-scan signature of Ag NPs of $5 \mathrm{~nm}$ (crossed red squares), $50 \mathrm{~nm}$ (crossed blue circles), and $200 \mathrm{~nm}$ (pointed green stars) for the pump wavelength of $532 \mathrm{~nm}$. The solid line is a fitting calculated using Eqs. 11 through 15 and parameters $z_{e}=0.007 \mathrm{~m}, z_{p}=0.2 \mathrm{~m}, d_{e}=1 \mathrm{~m}$, $D=1.4110^{-7} \mathrm{~m}^{2} / \mathrm{s}, \mathrm{k}=$ and $S_{o}=0.128$ for $5 \mathrm{~nm} \mathrm{Ag} \mathrm{NPs}$ (solid red line), $S_{o}=0.076$ for $50 \mathrm{~nm} \mathrm{Ag}$ NPs (solid blue line), and $S_{o}=0.0325$ for $200 \mathrm{~nm} \mathrm{Ag} \mathrm{NPs} \mathrm{(solid} \mathrm{green} \mathrm{line).}$ 
Figure 5. Absorption cross-sections as a function of the particle diameter measured using the PTL method for the pump wavelengths $405 \mathrm{~nm}$ (crossed blue squares), $532 \mathrm{~nm}$ (solid green stars), and $671 \mathrm{~nm}$ (crossed red circles). The black solid line represents a third order dependence. 


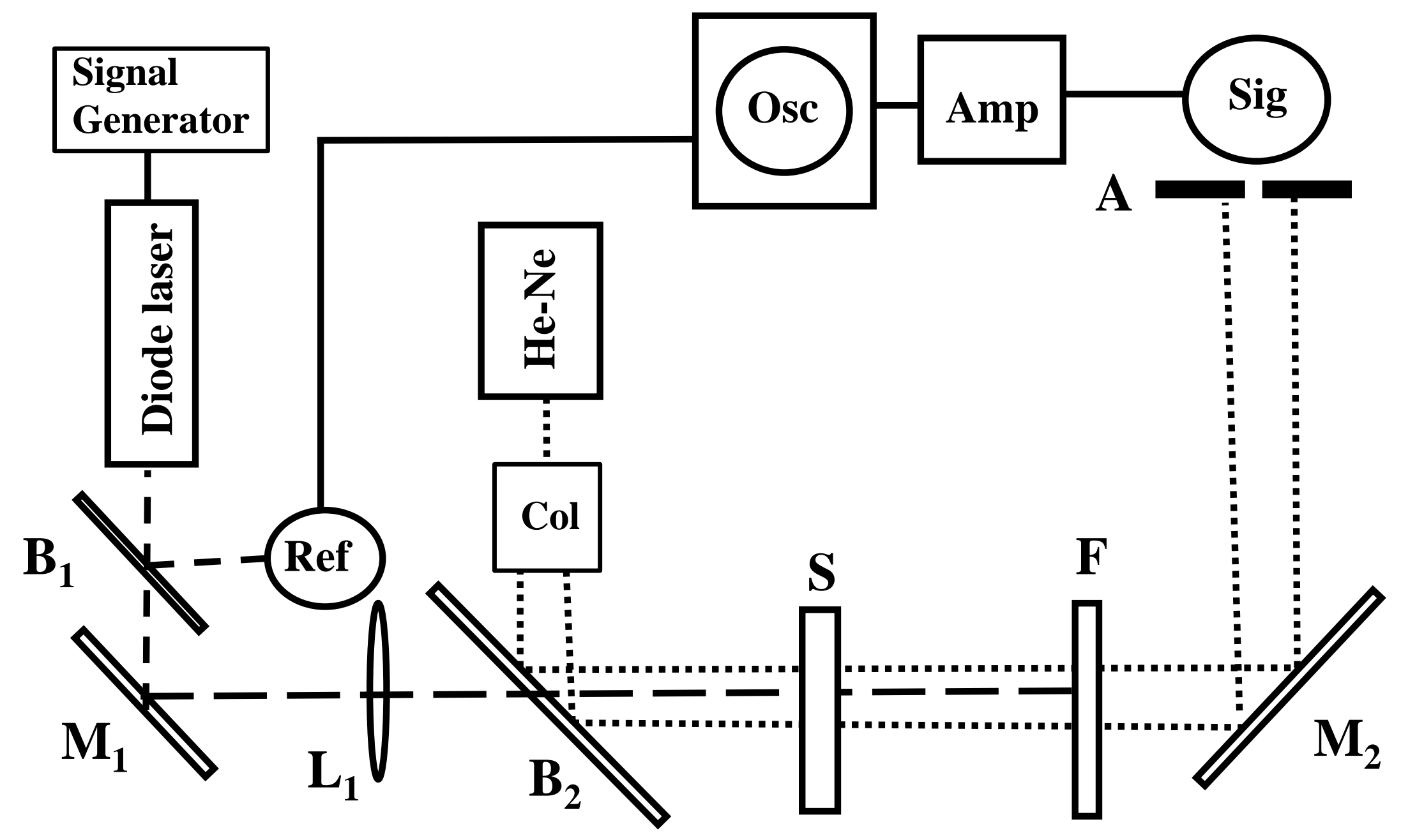




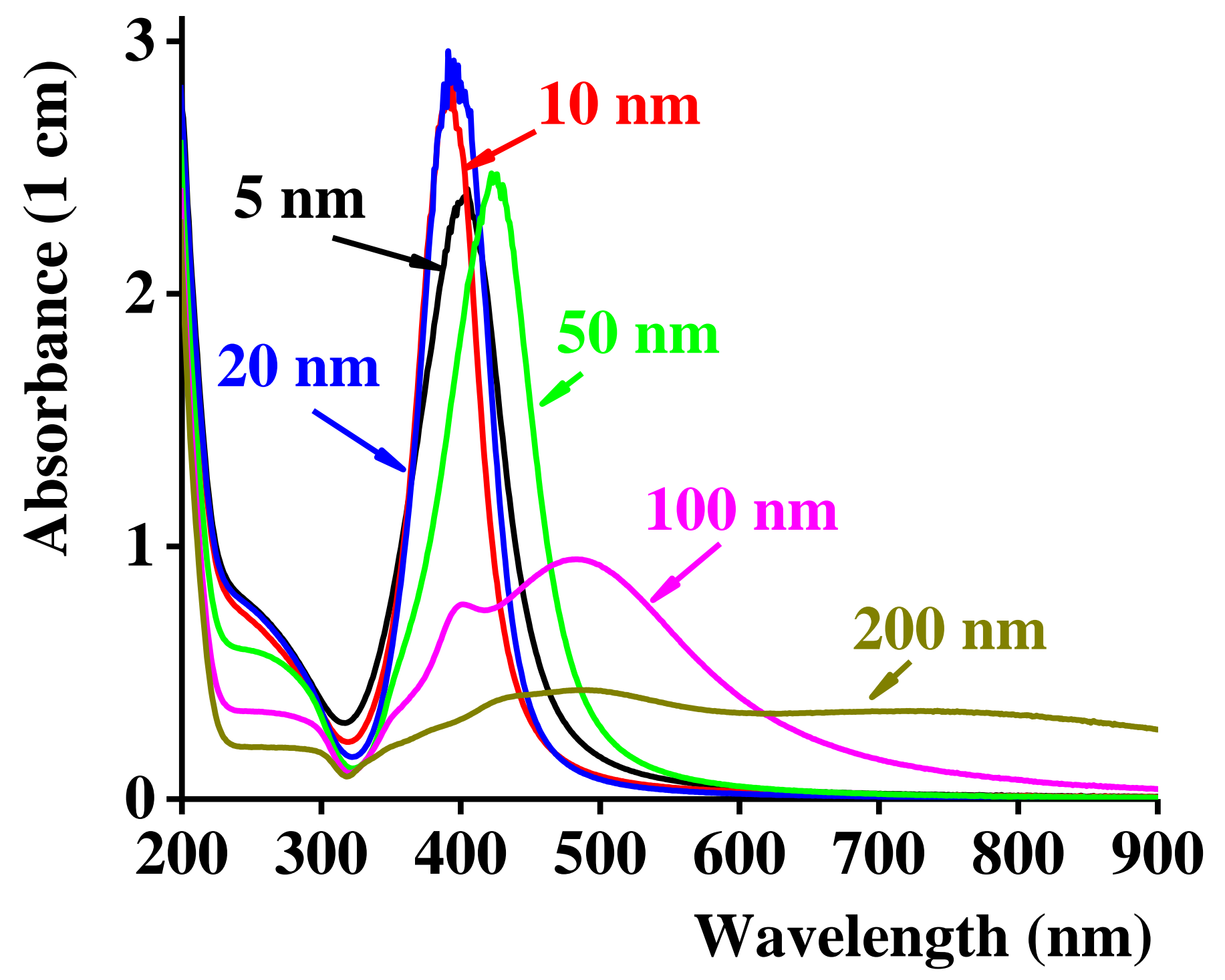



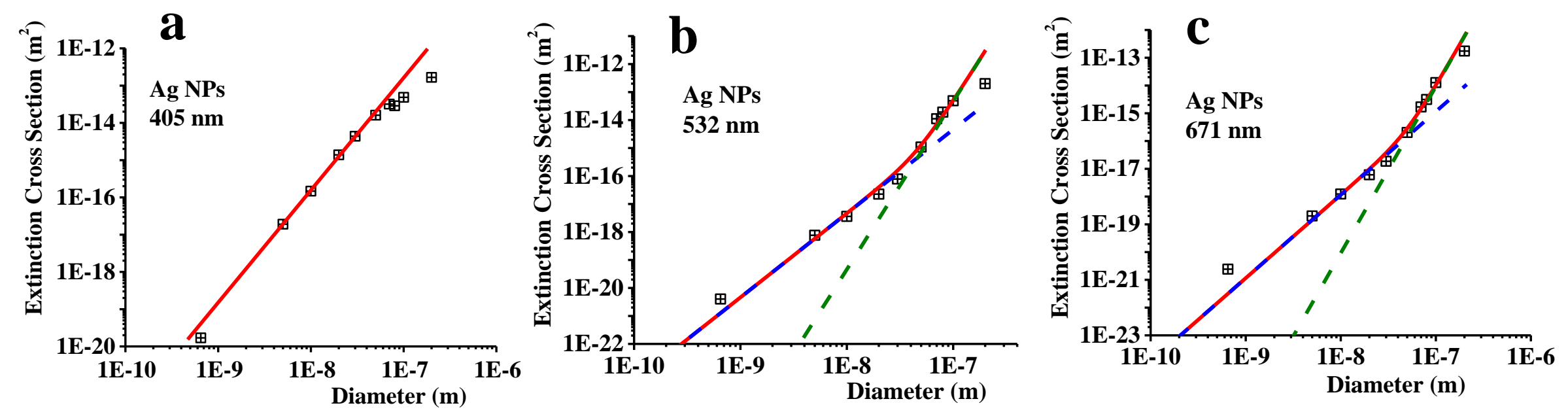


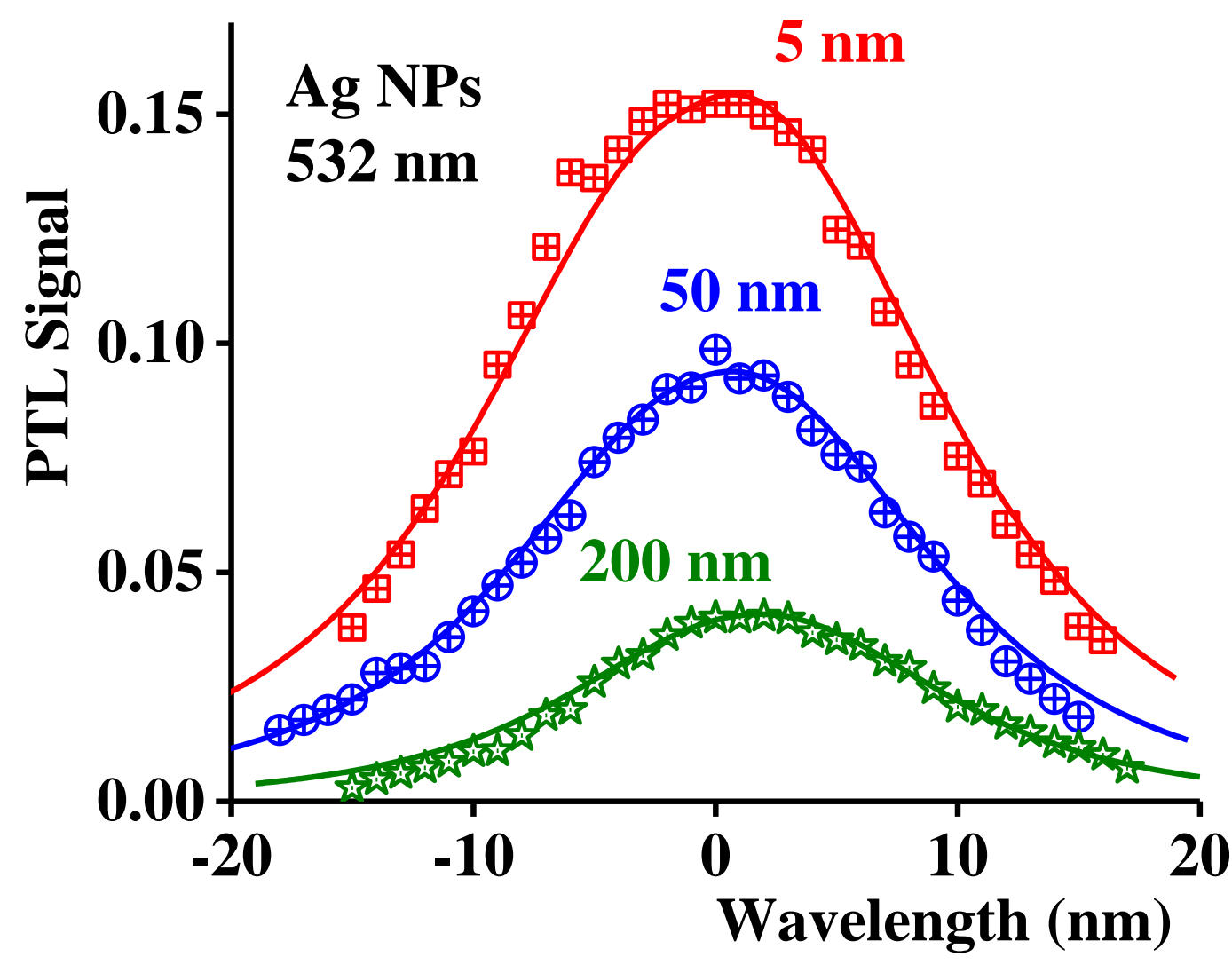




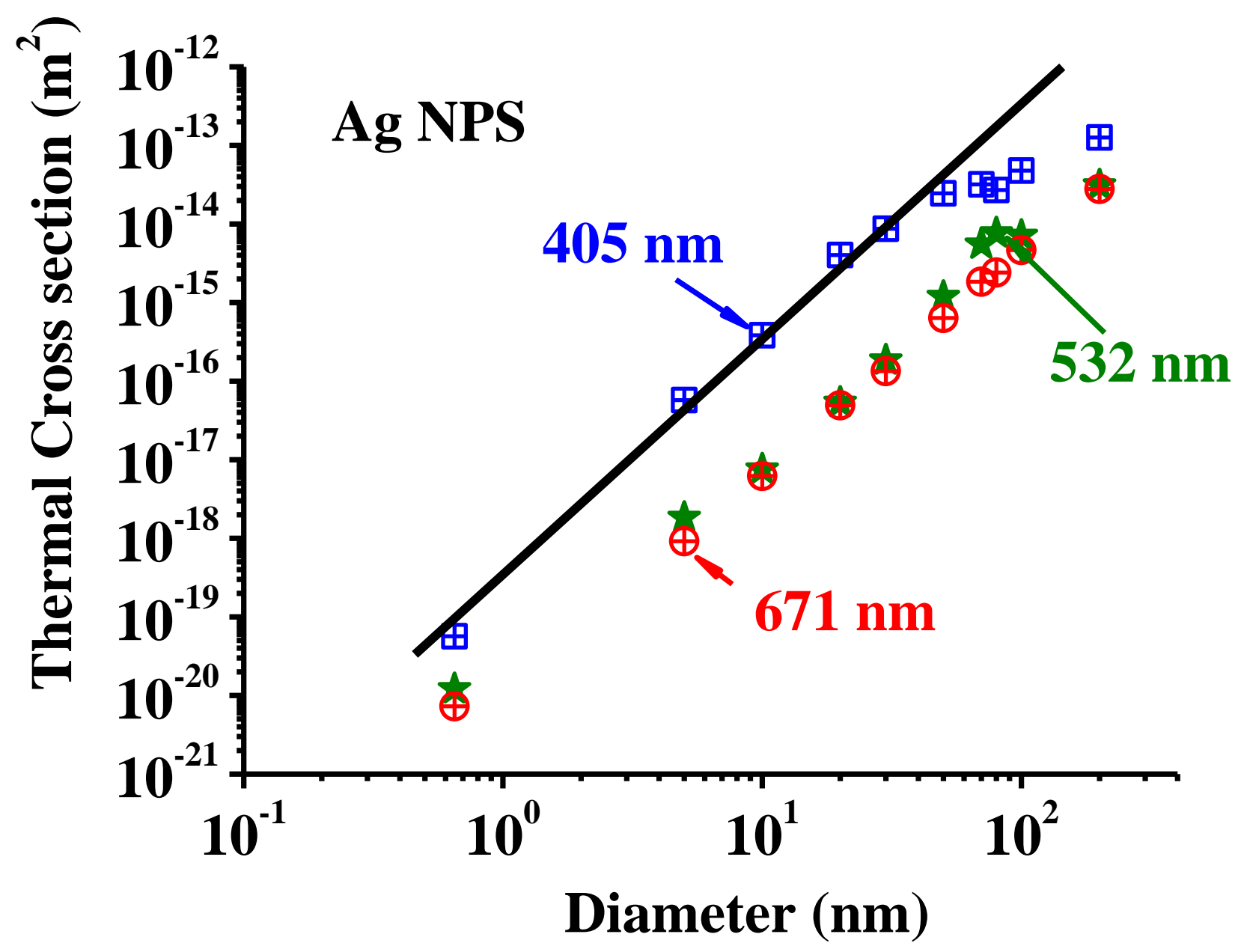

